\title{
Prediction of promoters and enhancers using multiple DNA methylation-associated features
}

\author{
Woochang Hwang ${ }^{1,5}$, Verity F Oliver ${ }^{1}$, Shannath L Merbs ${ }^{1}$, Heng Zhu ${ }^{2,3,4}$, Jiang Qian ${ }^{1,4^{*}}$ \\ From The International Conference on Intelligent Biology and Medicine (ICIBM) 2014 \\ San Antonio, TX, USA. 04-06 December 2014
}

\begin{abstract}
Background: Regulatory regions (e.g. promoters and enhancers) play an essential role in human development and disease. Many computational approaches have been developed to predict the regulatory regions using various genomic features such as sequence motifs and evolutionary conservation. However, these DNA sequence-based approaches do not reflect the tissue-specific nature of the regulatory regions. In this work, we propose to predict regulatory regions using multiple features derived from DNA methylation profile.
\end{abstract}

Results: We discovered several interesting features of the methylated $\mathrm{CpG}(\mathrm{mCpG})$ sites within regulatory regions. First, a hypomethylation status of CpGs within regulatory regions, compared to the genomic background methylation level, extended out $>1000$ bp from the center of the regulatory regions, demonstrating a high degree of correlation between the methylation statuses of neighboring $\mathrm{mCpG}$ sites. Second, when a regulatory region was inactive, as determined by histone mark differences between cell lines, methylation level of the $\mathrm{mCpG}$ site increased from a hypomethylated state to a hypermethylated state, the level of which was even higher than the genomic background. Third, a distinct set of sequence motifs was overrepresented surrounding $\mathrm{mCpG}$ sites within regulatory regions. Using 5 types of features derived from DNA methylation profiles, we were able to predict promoters and enhancers using machine-learning approach (support vector machine). The performances for prediction of promoters and enhancers are quite well, showing an area under the ROC curve (AUC) of 0.992 and 0.817 , respectively, which is better than that simply based on methylation level, especially for prediction of enhancers.

Conclusions: Our study suggests that DNA methylation features of $\mathrm{mCpG}$ sites can be used to predict regulatory regions.

\section{Introduction}

Transcriptional regulation plays an important role in most of biological processes. The interactions between transcription factors and regulatory regions, such as promoters and enhancers, are essential in transcriptional regulation. Therefore, identification of regulatory regions will provide mechanistic insight into various biological processes. Experimental and computational approaches have been developed to identify the regulatory regions on a genome-wide scale. For example, evolutionary

\footnotetext{
* Correspondence: jiang.qian@jhmi.edu

'Department of Ophthalmology, Johns Hopkins School of Medicine, Baltimore, MD, 21205 USA

Full list of author information is available at the end of the article
}

conservation, sequence motifs, and clustering of transcription factor binding motifs (or cis-regulatory modules) can be used to predict regulatory regions [1-4]. However, these approaches are based purely on DNA sequences, which do not reflect the tissue-specific nature of the regulatory regions.

Recently, histone marks were measured on a genomewide scale using the ChIP-seq technique [5-7]. These histone marks are good predictors for regulatory regions. For example, H3K4me1 is associated with active enhancers, while H3K4me3 is related to active promoters [8]. In addition, DNase I hypersensitivity sites (DHS) also localize to open chromatin regions, which are likely regulatory regions $[9,10]$. The ENCODE project has generated 
histone marks and DHS profiles in multiple cell lines and tissues $[8,9,11,12]$, which provide valuable information to understand the organization and dynamics of the regulatory regions in the cells.

In this work, we propose to predict regulatory regions by utilizing the DNA methylation patterns. DNA methylation, the addition of a methyl group to the fifth carbon of a cytosine residue adjacent to a guanine (a CpG site), is a well-studied epigenetic modification. While DNA methylation is considered stable and heritable in differentiated somatic cells, it can also change dynamically during the lifespan of a cell and is susceptible to diet and other environmental influences $[13,14]$. DNA methylation is known to play a role in gene regulation. It is well accepted that DNA methylation in promoter regions represses the expression of the genes [15]. High-throughput technologies, such as whole genome bisulfite sequencing and array-based methods, have enabled the mapping of DNA methylation patterns on a genomic scale, identifying hundreds of millions of methylated cytosines [16-19].

DNA methylation-based approach has been developed to predict regulatory regions in recent work [20,21]. In these studies, low methylation regions are associated with distal regulatory elements, as they are enriched for active histone marks (e.g. H3K4me1), DNase-hypersensitive sites, and transcription factor binding sites (TFBS). In this study, we performed a comprehensive survey of $\mathrm{mCpGs}$ in regulatory regions and explore whether we can extract a set of DNA methylation dependent features besides the methylation level to improve the prediction. By examining these properties of the mCpG sites across different cell lines, we discovered that these sites did demonstrate specific genomic properties. Using these genomic features, we were able to predict regulatory regions using support vector machine approach. The paper is organized as follows. We first defined the positive and negative sets for regulatory region prediction. We then described the novel features derived from DNA methylation profiles. Finally, we utilized machine-learning approach (support vector machine) to predict regulatory regions (promoters and enhancers separately) based on the features we obtained. Our results demonstrate that the performance of the prediction based on multiple DNA methylation-associated features is better than the prediction solely based on methylation level.

\section{Results}

\section{Selection and assessment of positive and negative} datasets

We used the previously established definition of a regulatory region as determined by genome-wide histone modification signatures [8]. For example, H3K4me3 is known to be associated with promoters, while H3K4me1 with enhancers. Ernst et al. predicted different types of regulatory regions using a hidden Markov model. In this paper, we focus on two major regulatory regions: promoters and enhancers.

We also selected the same numbers of the $\mathrm{mCpG}$ sites in random genomic regions as negative datasets. To exclude the effect of differential methylation due to genomic location, we chose random genomic regions with the same relative distance to the nearest transcription start site (TSS) or exon-intron boundary for each type of regulatory regions (see Methods for details).

To determine the quality of the regulatory regions determined by histone marks, we calculated the correlation between the methylation level of a $\mathrm{mCpG}$ site and the expression of its associated target genes (Figure 1A). The adjacent gene of a promoter was selected as its target gene. The predicted target genes for enhancers were obtained from Thurman et al. [9], which is based on the correlation of DNase I hypersensitivity activity between distal elements and promoters across cell lines. For each methylation site, Pearson correlation coefficient was used to compute the correlation between methylation level at a given site and the expression of its target gene across cell lines [22]. As comparison, the same correlation was calculated for the negative datasets.

Compared to the $\mathrm{mCpG}$ sites in random genomic regions, the methylation level of the $\mathrm{mCpG}$ sites in the regulatory regions was significantly more negatively correlated with the expression of the target genes $(\mathrm{p}<$ 1.0E-15; Kolmogorov-Smirnov test) (Figure 1B and Figure 1C). This is consistent with the notion that DNA methylation represses gene expression. We would like to point out that the conclusion still holds if we used Spearman's rank correlation, rather than Pearson correlation coefficient (Additional file 1: Figure. S1A, B).

Overall, our result suggests that the regulatory regions predicted by histone marks and the random genomic regions with similar relative genomic locations are of reasonable quality and are suitable to serve as the positive and negative sets of this study.

\section{mCpGs in regulatory regions are hypomethylated}

We then extract a series of DNA methylation-dependent features by comparing the methylation sites in the regulatory regions and random $\mathrm{mCpG}$ sites in the negative sets. We found that the methylation level of regulatory $\mathrm{mCpG}$ sites was significantly lower than the other mCpG sites (Figure 2). The average methylation levels of $\mathrm{mCpGs}$ in active promoters and the corresponding negative set are 0.34 and 0.72 , respectively, showing statistically significant difference $(\mathrm{p}<1.0 \mathrm{E}-15$ based on Kolmogorov-Smirnov test). Similarly, the methylation levels of mCpGs in strong enhancers and the corresponding negative sets are 0.69 and 0.84 , respectively ( $\mathrm{p}<1.0 \mathrm{E}-15$ based on Kolmogorov-Smirnov test). These results persisted when $\mathrm{CpG}$ islands in the regulatory 


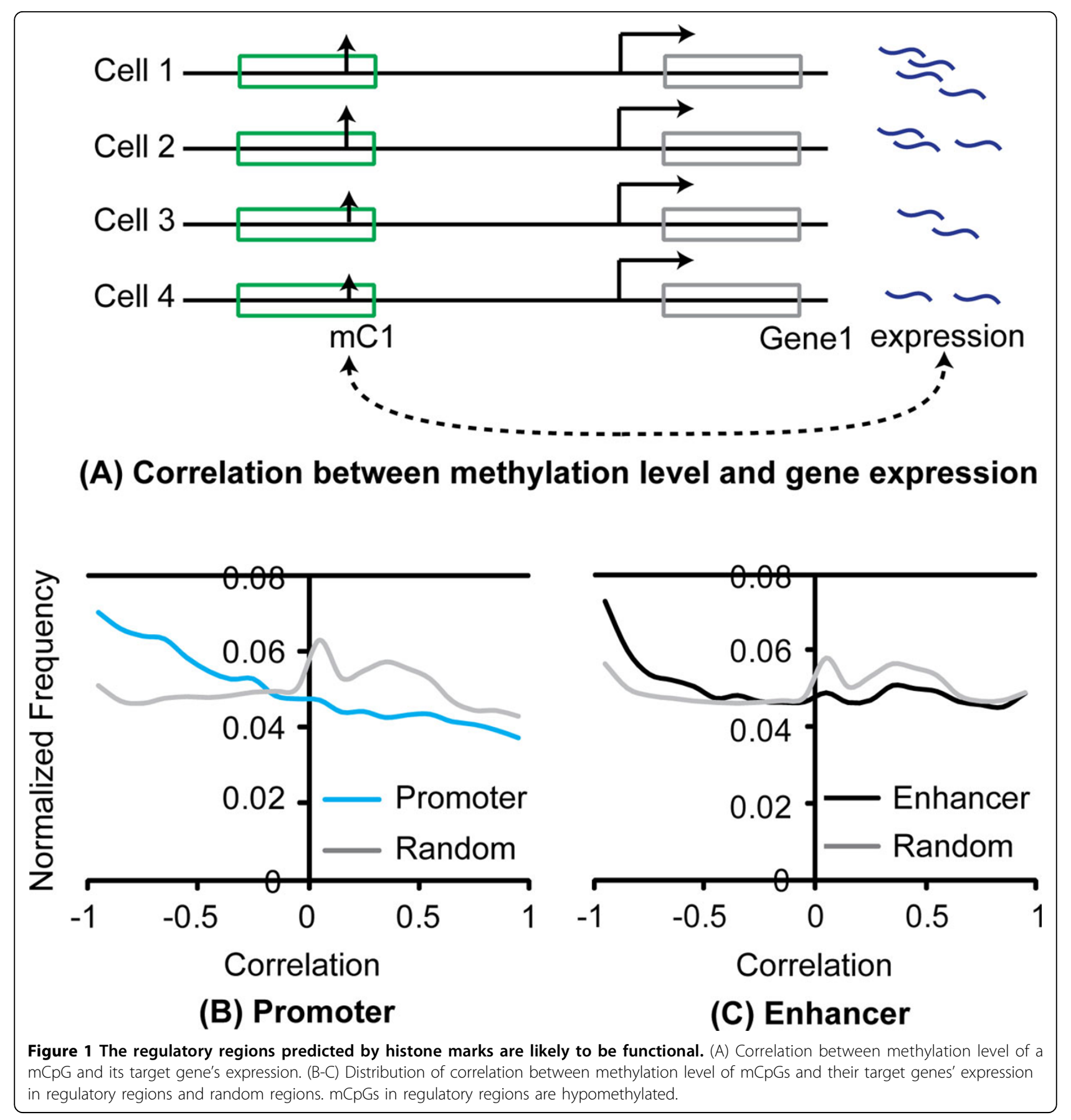

regions and the corresponding random regions were excluded, indicating that the low methylation level in regulatory regions was not due to the overlap with $\mathrm{CpG}$ islands, which are generally hypomethylated (Figure 2B, $2 \mathrm{C})$. Interestingly, regulatory regions overlapping with CpG islands demonstrated lower methylation levels than regulatory regions outside of the CpG islands ( $\mathrm{p}<1.0 \mathrm{E}-15$ based on Kolmogorov-Smirnov test; Figure 2B, 2C), suggesting that those $\mathrm{CpG}$ islands playing a regulatory role have lower methylation levels. Overall, our observations suggested that hypomethylation is a key feature of regulatory regions, independent of the relative location to a $\mathrm{CpG}$ island.

Hypomethylation in regulatory regions extends across a long range

Within a methylated regulatory region, the lowest methylation level is found centrally within the region, 


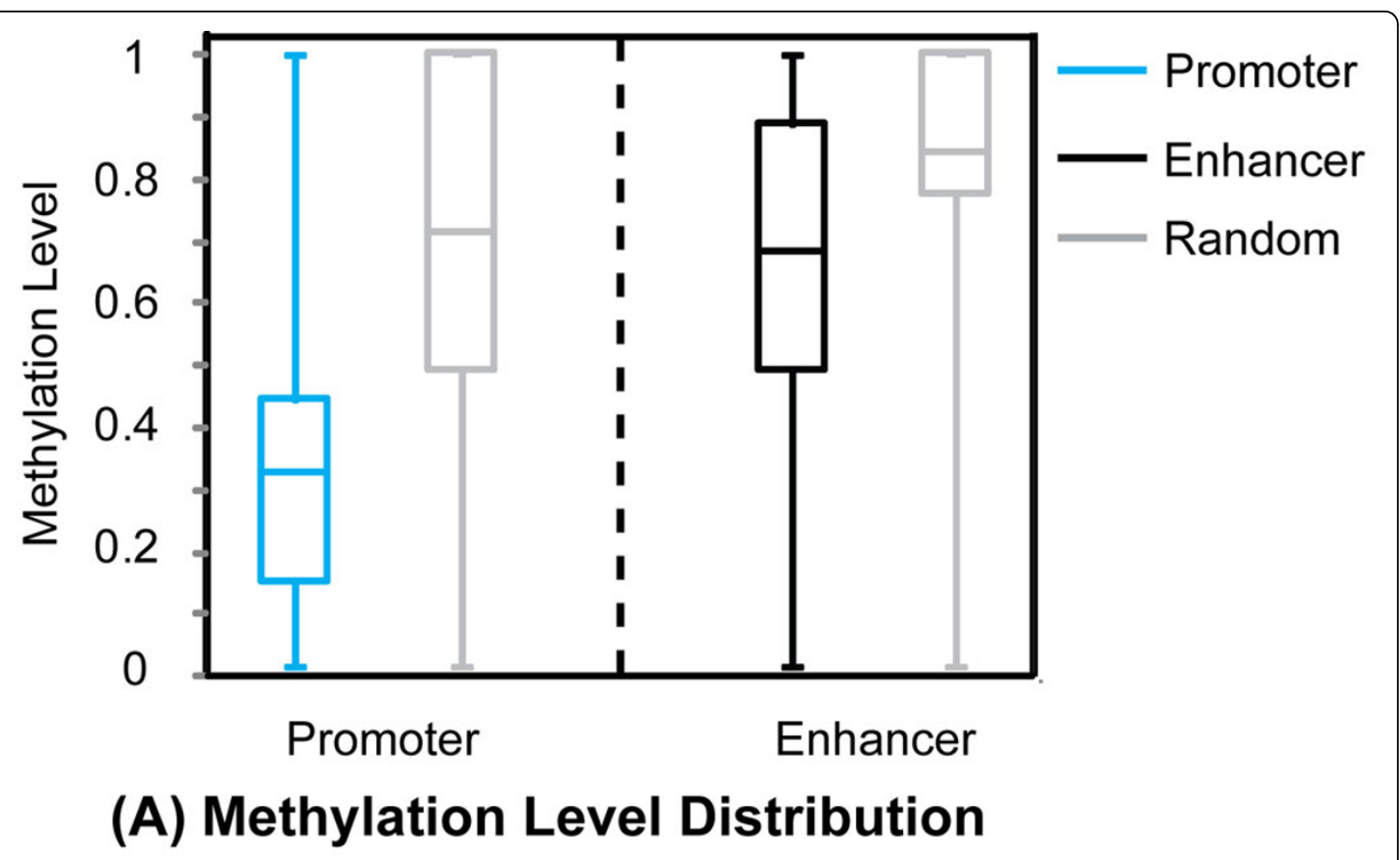

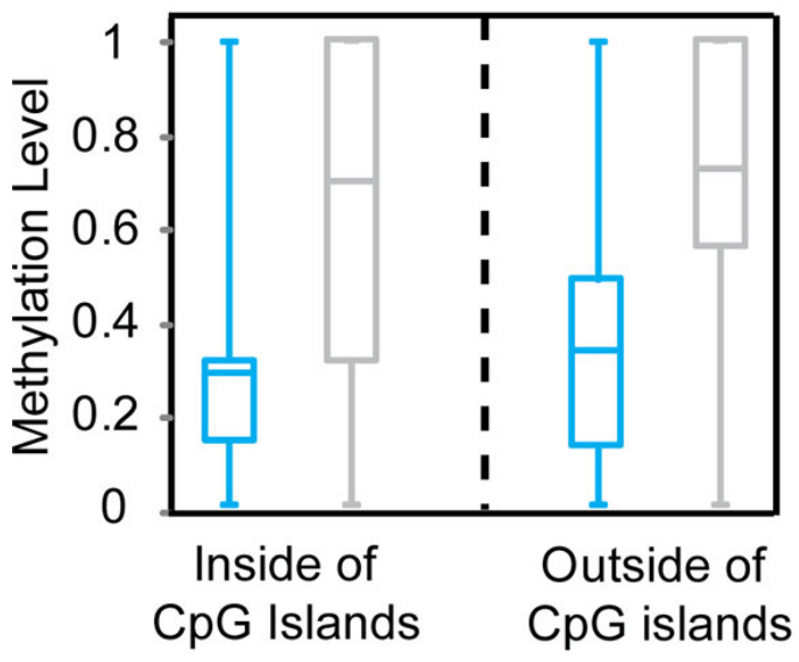

(B) Promoter

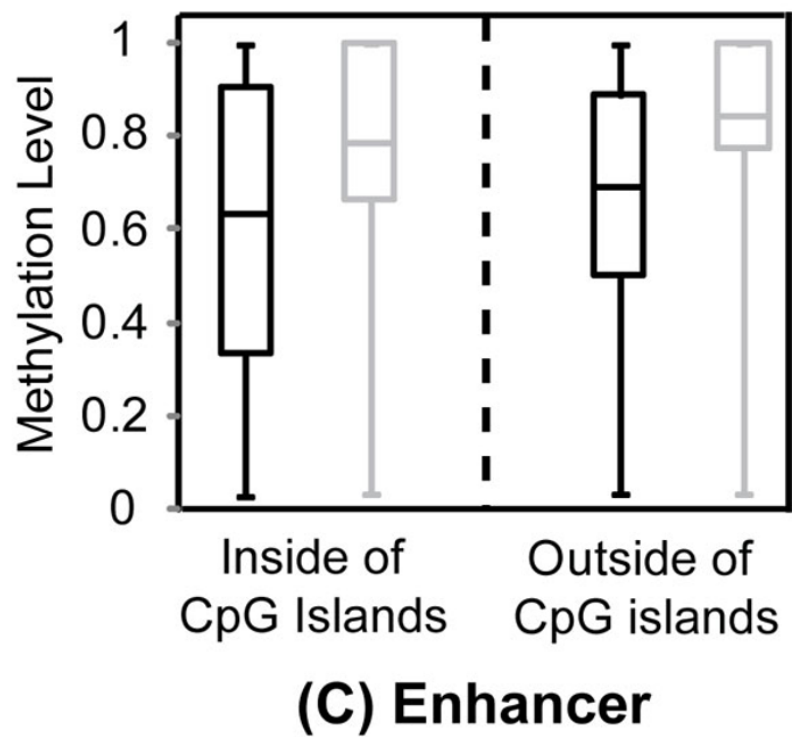

Figure 2 CpG sites are hypomethylated in regulatory regions. (A) Methylation level distribution of mCpGs in regulatory regions (blue and black) and random regions (grey). (B-C) Methylation level distribution of $\mathrm{mCpGs}$ inside and outside of $\mathrm{CpG}$ islands for regulatory regions (blue and black) and random regions (grey).

and this hypomethylation extends across a long range (Figure 3). For example, the average methylation level of mCpGs at the center of active promoters (0.34) gradually increases across the promoter region and reaches a plateau (0.82) at $\sim 1500$ bp away, suggesting that hypomethylation is not localized to a narrow genomic region (Figure 3A). The extended hypomethylated regions were also apparent within strong enhancers (Figure 3B).
Strikingly, the extended hypomethylation was also observed within transcription factor (TF) binding sites, which are generally more localized to $10-20$ bp regions. Using ChIP-seq and predicted data for several TFs [23], we found that the methylation levels were lowest in the center of TF binding sites and reached background level by $1500 \mathrm{bp}$ (Figure 3C), despite the fact that the majority of binding sites are less than $20 \mathrm{bp}$ in length. 


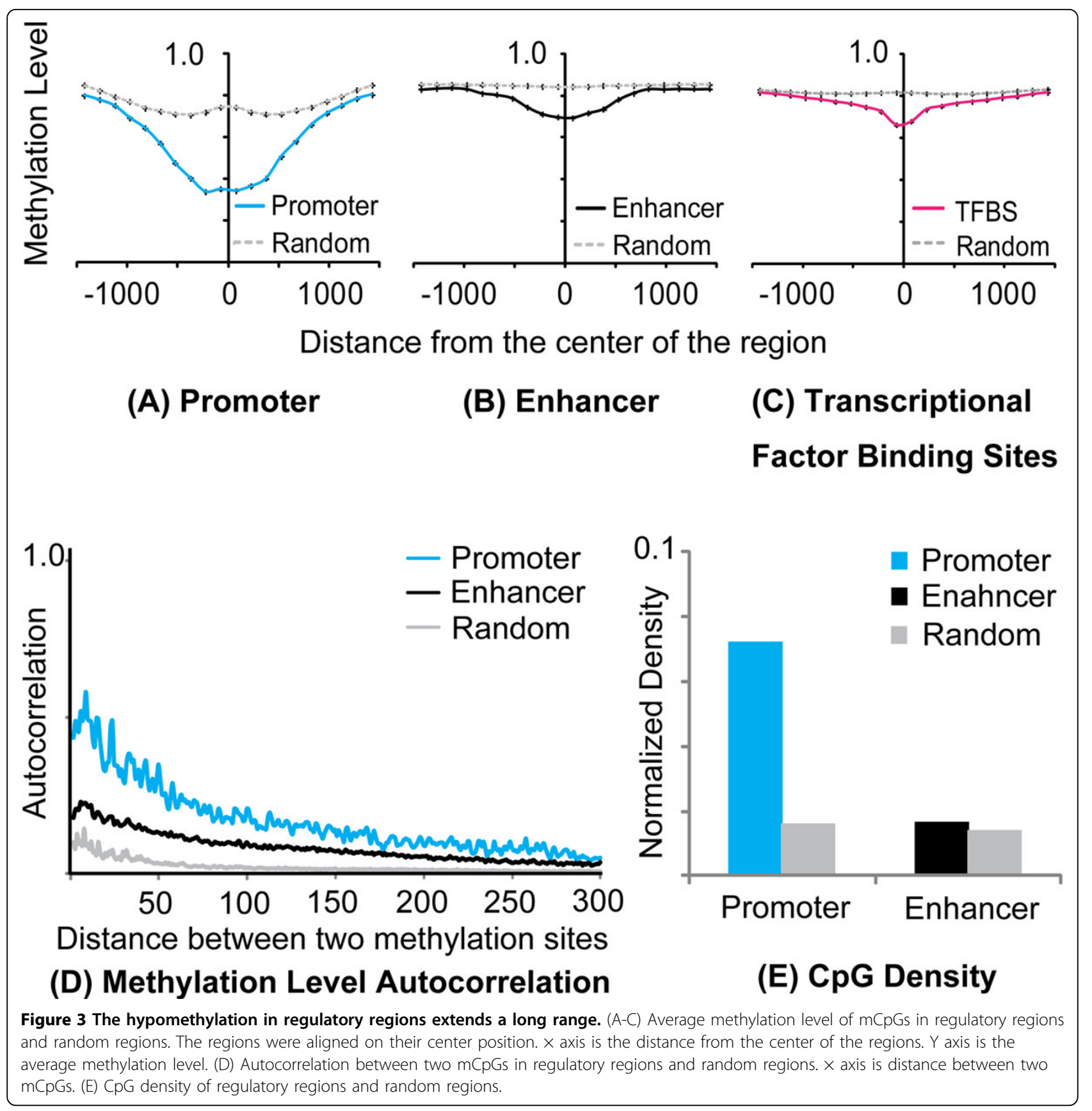

Methylation levels of neighboring regulatory $\mathrm{mCpG}$ sites are highly correlated

One explanation of the extended hypomethylation is correlation between neighboring regulatory $\mathrm{mCpG}$ sites. To test this hypothesis, we calculated the autocorrelation of methylation profiles within regulatory regions. Specifically, we computed the correlation of methylation levels at two methylation sites as a function of their genomic distances (Figure 3D, see Methods for detail). As expected, $\mathrm{mCpG}$ sites within close proximity showed higher correlation than those located distally. Based on the autocorrelation of methylation profile, we observed that the correlation of methylation between $\mathrm{mCpG}$ sites was significantly stronger in regulatory regions than in non-regulatory regions $(\mathrm{p}<$ 1.0E-15 based on Kolmogorov-Smirnov test; Figure 3D). In regulatory regions, the correlation of methylation levels extended across distances of up to 326 and 231bp for promoters and enhancers, respectively, whilst in random genomic regions the correlation disappeared by $41 \mathrm{bp}$ (see also Additional file 1: Figure S1C).

We speculated that a high density of $\mathrm{CpG}$ sites could be the underlying mechanism for correlation between 
methylation levels, as sites in close proximity might be co-regulated by DNA methyltransferases. We examined the CpG densities in the regulatory and random regions and found that regulatory $\mathrm{CpG}$ sites were much denser than those in random regions, especially within active promoters (Figure 3E). This observation was true even if the $\mathrm{CpG}$ islands in these regions were excluded (Additional file 1: Figs S1D, E). Thus, the hypomethylation in regulatory regions and/or TF binding sites can be attributed to both the high correlation between methylation levels and the high CpG density within these regions.

\section{Inactive regulatory regions are hypermethylated}

Epigenetic marks in regulatory regions differ between different cell types. For example, in a given cell line a genomic region can be an active promoter enriched for active histone marks (e.g. H3K4me3, H3K9ac, and $\mathrm{H} 3 \mathrm{~K} 27 \mathrm{ac}$ ), while the same region may be inactive in another cell line due to the absence of such histone marks. To address the role of histone modifications within regulatory regions, we compared changes in the methylation level of the regulatory $\mathrm{mCpG}$ sites to changes in histone mark status. The methylation levels between two cell lines (H1 and GM12878) were examined, as genome-wide histone modification and methylation data were available for both cell lines. We defined three genomic regions for each cell line: transcriptionally active, transcriptionally inactive, and random (background) regions. For example, the genomic regions predicted as regulatory regions in $\mathrm{H} 1$ cell were defined as 'active' in $\mathrm{H} 1$ cell. The genomic regions that were predicted as regulatory regions in GM12878 cell, but not in $\mathrm{H} 1$ cell were categorized as 'inactive' in $\mathrm{H} 1$ cell. These "inactive" regions represent the genomic regions that are not enriched by transcriptionally active histone marks in $\mathrm{H} 1$, but have the potential to become transcriptional active in other cell lines. As comparison, we also selected a set of genomic regions that do not overlap with any regulatory region in both $\mathrm{H} 1$ and GM12878 (see details in Methods and Figure 4A). The mCpGs methylation levels inside active, inactive, and random regions in $\mathrm{H} 1$ cell were compared.

Using our comparative analysis between $\mathrm{H} 1$ and GM12878 cells, we found that the methylation levels at $\mathrm{mCpG}$ sites within inactive promoters and enhancers resembled neither the active nor the background (random region) levels (Figure 4B). Interestingly, the methylation levels in inactive regulatory regions were even higher than background levels obtained from random regions $(\mathrm{p}<$ 1.0E-15; Kolmogorov-Smirnov test) (Figure 4B). This phenomenon was robust as it was present when we compared H1 cells with other different cell lines (additional file: 2 : Figure S2 A-E). Furthermore, the finding still held if we grouped the regulatory regions based on whether they overlap with $\mathrm{CpG}$ islands (Figure 4B, additional file 2: Figs S2 A-E, 3: Figure. S3). Finally, rather than using predicted active and inactive regulatory regions, if we used the raw histone marks (e.g. H3k9ac), we made the same observation in multiple cell lines (Additional file 4: Figure S4). Our findings demonstrate that inactive regulatory regions show hypermethylation relative to the genomic background, distinguishing inactive regulatory regions from background methylation.

\section{Methylation level in regulatory regions can vary considerably between cells}

The difference in methylation levels between active and inactive regions suggested that regulatory $\mathrm{mCpG}$ sites might have a greater range of possible methylation levels than other mCpGs. To test this hypothesis, we calculated the variability of each $\mathrm{mCpG}$ site across the cell lines whose genome-wide methylation profiles are publically available [22]. The variance of the methylation levels for the regulatory regions predicted in $\mathrm{H} 1$ cells were then compared to those from random genomic regions (Figure $4 \mathrm{C}, 4 \mathrm{D}$ ). About $40 \%$ of the regulatory $\mathrm{mCpG}$ sites have a variance larger than 0.2 , whereas only $15 \%$ of $\mathrm{mCpG}$ sites in other genomic regions showed the same variance, suggesting that the regulatory $\mathrm{mCpG}$ sites have a larger range of potential methylation levels.

We then directly related the methylation level of $\mathrm{mCpGs}$ to the regulatory state (active versus inactive). For those $\mathrm{mCpG}$ sites that were present in an active regulatory region in both cell lines, the difference in methylation level between the two cell lines was small (Additional file 2: Fig. S2F). In fact, the vast majority $(\sim 75 \%)$ of the regulatory $\mathrm{mCpG}$ sites showed very small methylation level differences in the range of -0.1 to 0.1 between the two different cell lines. This difference was similar to the difference in methylation levels of $\mathrm{mCpG}$ sites in random genomic regions. In contrast, the regulatory $\mathrm{mCpG}$ sites present in both active and inactive regulatory regions in two cell lines showed a greater methylation difference; less than a half (42\%) of the active-inactive regulatory regions had methylation level differences between -0.1 and 0.1 . Taken together, the large range of potential methylation levels in the regulatory regions can be associated with the status changes of the regulatory regions.

\section{Distinct sequence motifs are associated with regulatory $\mathrm{mCpG}$ sites}

Since DNA motifs were used to predict enhancers, we also explore to identify the DNA motifs associated with $\mathrm{mCpG}$ sites in regulatory regions. The rationale is that DNA methylation is believed to play a crucial role in the transcription process by directly interfering with TF binding to 


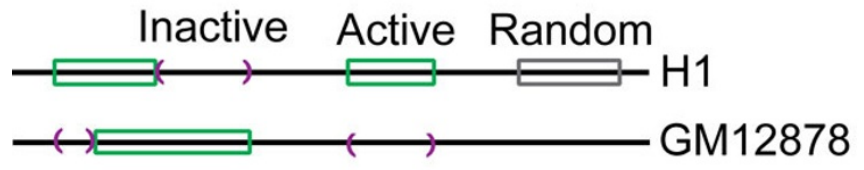

(A) Regulatory Region Functional Status

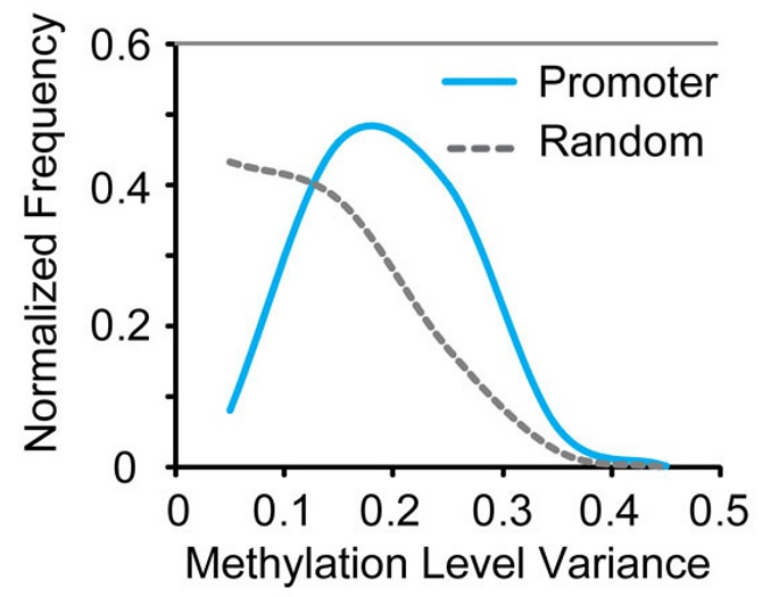

(C) Promoter

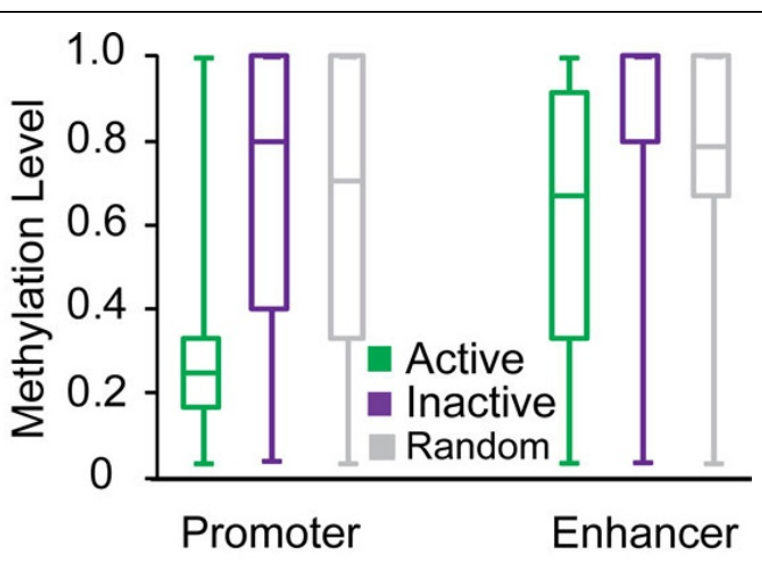

(B) Methylation Level

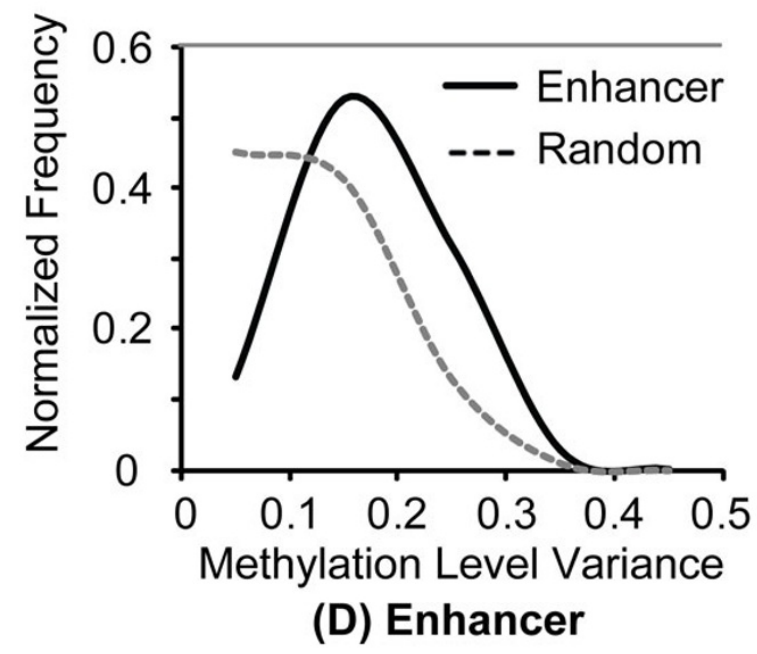

Figure 4 Methylation level changes with the regulatory region status. (A) Functional status changes of a genomic region among different cells. A region can be active (green) in a cell ( $\mathrm{H} 1)$ while inactive (purple) in another cell (Gm12878). (B) Methylation level distribution of mCpGs inside of $\mathrm{CpG}$ islands in active, inactive and random regions. (C-D) Methylation level variance of $\mathrm{mCpGs}$ in regulatory and random regions among 15 cell lines.

the regulatory regions [24]. Therefore, we hypothesize that sequence motifs around the $\mathrm{mCpGs}$ in regulatory regions were more likely to be TF binding sites and would serve as another informative feature distinguishing regulatory mCpG sites. We examined all 8-mer sequences with a $\mathrm{CpG}$ in the center and compared the occurrence of each motif in the regulatory regions and in the random regions. The statistical significance of each motif was evaluated, and both overrepresented and underrepresented motifs were identified. In total, 86 and 194 8-mers were significantly overrepresented in the active promoters and the strong enhancers, respectively, compared to background ( $\mathrm{p}<1.0 \mathrm{E}-5)$. Additionally, we found 104 and 86 significantly underrepresented 8-mers in the active promoter and the strong enhancers $(\mathrm{p}<1.0 \mathrm{E}-5)$. Figure 5 lists the top 5 most significantly overrepresented and underrepresented 8-mers in the regulatory regions (see additional file 5: Fig. S5 for additional motifs). These motifs were also compared with known transcription factor consensus sequences (5: Figure. S5).

Interestingly, the nucleotide composition of the overrepresented 8-mers was different from that of the underrepresented 8-mers. For example, the GC content of overrepresented 8-mers obtained from the promoters and enhancers are 0.68 and 0.60 , respectively. In contrast, the GC contents of the underrepresented 8-mers from the promoters and enhancers are only 0.47 and 0.31 , respectively. This distinct nucleotide composition difference became more significant when considering only the 2 bases directly adjacent to the CpG sites. Over $77 \%$ and $68 \%$ of the overrepresented 8 -mers in the promoters and enhancers, respectively, had either cytosines 


\begin{tabular}{|c|c|c|c|}
\hline Motif & p-value & Logo & TF \\
\hline CTCCGCCC & $<<1.0 \mathrm{E}-15$ & $5 \mathbf{G}_{-}$ & SP1 \\
\hline CTCCGAGC & $<<1.0 \mathrm{E}-15$ & 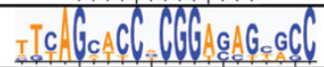 & NRSE \\
\hline CGCCGCCG & $<<1.0 \mathrm{E}-15$ & 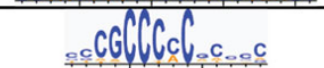 & KROX \\
\hline CGCCGCCC & $<<1.0 \mathrm{E}-15$ & GGf $\mathrm{CGG}_{\mathrm{GG}}$ & SP1 \\
\hline GGGCGGAA & $<<1.0 \mathrm{E}-15$ & 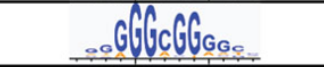 & SP1 \\
\hline \multicolumn{4}{|c|}{ Overrepresented } \\
\hline Motif & p-value & Logo & TF \\
\hline ACACGAGC & $<<1.0 \mathrm{E}-15$ & 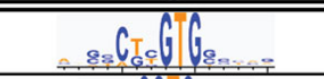 & HES1 \\
\hline ACACGTAC & $<<1.0 \mathrm{E}-15$ & 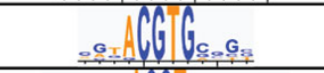 & HIF1 \\
\hline ACACGTCT & $<<1.0 \mathrm{E}-15$ & $\therefore \quad \operatorname{Cog} G$ & XBP1 \\
\hline GAACGTGA & $<<1.0 \mathrm{E}-15$ & 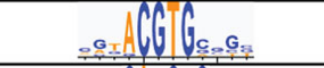 & HIF1 \\
\hline ACACGTGA & $<<1.0 \mathrm{E}-15$ & $\operatorname{Cis}_{5} \log _{0}$ & USF2 \\
\hline
\end{tabular}

Underrepresented

(A) Active Promoter

\begin{tabular}{|c|c|c|c|}
\hline Motif & p-value & Logo & TF \\
\hline ACACGCAC & $<<1.0 \mathrm{E}-15$ & $\int C[G] G_{-}$ & AHR \\
\hline CAGCGTGG & $<<1.0 \mathrm{E}-15$ & 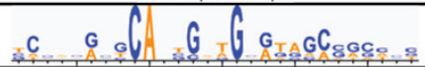 & PAX5 \\
\hline CCACGGAG & $<<1.0 \mathrm{E}-15$ & 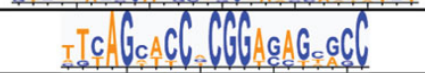 & NRSE \\
\hline AGCCGCAG & $<<1.0 \mathrm{E}-15$ & 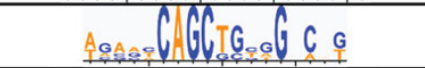 & AP4 \\
\hline CAGCGTGA & $<<1.0 \mathrm{E}-15$ & $\mathrm{Ag}_{\mathrm{G}} \mathrm{TG}_{\mathrm{A}}$ & PAX8 \\
\hline \multicolumn{4}{|c|}{ Overrepresented } \\
\hline Motif & p-value & Logo & TF \\
\hline ACGCGATC & $<<1.0 \mathrm{E}-15$ & 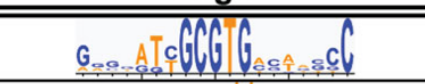 & $\overline{\text { AHRARNT }}$ \\
\hline CATCGATA & $<<1.0 \mathrm{E}-15$ & 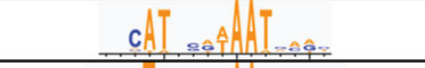 & BRN2 \\
\hline TATCGTAA & $<<1.0 \mathrm{E}-15$ & $G \quad I G, G c h=c A$ & CEBP \\
\hline TAGCGTGA & $<<1.0 \mathrm{E}-15$ & 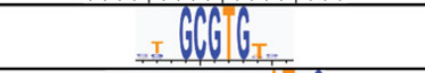 & AHR \\
\hline AATCGTAT & $<<1.0 \mathrm{E}-15$ & CACcaAta & CDPCR3 \\
\hline
\end{tabular}

(B) Strong Enhancer

Figure 5 DNA Motifs are associated with CpG sites in regulatory regions. 5 most significantly overrepresented and underrepresented sequence motifs surrounding $\mathrm{mCpGs}$ in regulatory regions. Third and fourth column show the sequence logos and the names of the best matched transcriptional factor binding sites. (A) Active promoter (B) Strong enhancer

or guanines as a direct neighbor of the CpG site. In contrast, only $31 \%$ and $28 \%$ of the underrepresented 8 mers in these two types of regulatory regions had either cytosines or guanines at these positions. Cytosines or guanines were simultaneously observed in both direct neighbors of the overrepresented motifs (56\% and $45 \%$ in the promoters and enhancers, respectively), which occur much less frequently in the underrepresented 
motifs $(13 \%$ and $11 \%$ in the promoters and enhancers, respectively).

\section{Regulatory regions are predictable}

Distinct genomic features of the regulatory $\mathrm{mCpGs}$, which distinguish them from other $\mathrm{mCpGs}$ in negative sets, were used to predict regulatory regions. We predicted the regulatory regions using these features, including methylation level, $\mathrm{CpG}$ density, autocorrelation of methylation levels, variance of methylation levels among different cell lines and sequence motifs (significance (-log $(P))$ of the occurrence of 8 -mer sequence motifs surrounding the $\mathrm{mCpGs}$ ). The computation of these features is described in detail in Methods section. As comparison, we predicted the regulatory regions solely based on methylation level.

Support vector machine (SVM) was used to classify regulatory and non-regulatory regions. SVM finds a set of hyper planes that separates data points into a set of classes in high dimensional feature space. To distinguish between regulatory and random genomic regions, 10 -fold cross validation was used to measure the classification performance, in which $90 \%$ of regions were used to train the model, and the remaining $10 \%$ of regions were used as test dataset (see details in Methods). Figure 6A illustrated the performance of the prediction for active promoters and strong enhancers using a Receiver Operating Characteristic (ROC) curve. The area under the ROC curve (AUC) was used to depict the performance of a binary classification. Active promoters and strong enhancers were predicted quite well, showing an AUC of 0.992 and 0.817, respectively (Figure 6A). Perhaps not surprisingly, if we only used the methylation levels as the feature for prediction, the performance is lower, with AUC of 0.985 and 0.692 for promoters and enhancers, respectively. The prediction for enhancers is significantly improved with the additional features.

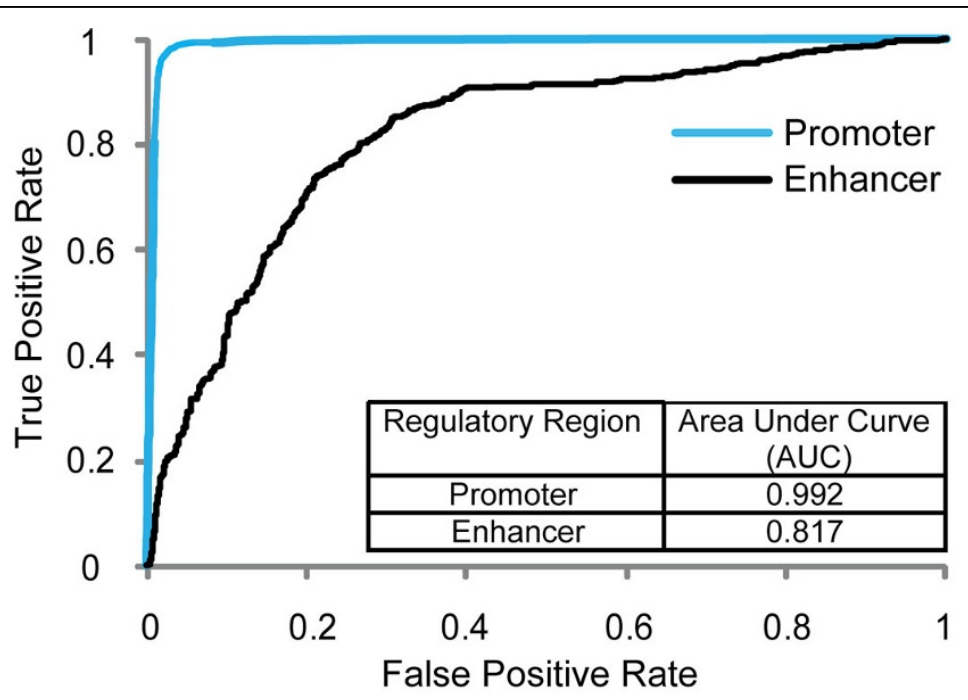

(A) Receiver operating characteristic curve (ROC)

\begin{tabular}{|l|c|c|}
\hline \multirow{2}{*}{ Feature } & \multicolumn{2}{c|}{ Information Gain } \\
\cline { 2 - 3 } & Promoter & Enhancer \\
\hline Methylation Level & 0.71 & 0.21 \\
\hline Methylation Variance & 0.67 & 0.06 \\
\hline CpG Density & 0.59 & 0.42 \\
\hline Methylation Auto CC & 0.48 & 0.26 \\
\hline Sequence Motif P-value & 0.16 & 0.04 \\
\hline
\end{tabular}

(B) Information Gain

Figure 6 Regulatory regions can be predicted using the features derived from DNA methylation profiles. (A) Receiver operation characteristic curve (ROC) of regulatory region prediction using methylation profile in the regions. Support vector machine (SVM) was used. (B) Information gain of features in the prediction. 
Some features showed more significant contribution to the prediction than other features. Therefore, the ability of each feature to discriminate regulatory and non-regulatory regions was analyzed by the information gain of each feature (Figure 6B). Information gain (IG) of a given feature $F$ with respect to the classes (e.g., regulatory or random regions) is the entropy reduction of the sample set when we know the feature $F$ (see Methods in detail). Interestingly, the most informative features are different when predicting promoters and enhancers. For the promoters, the features showing the largest information gain are methylation level and methylation variance, while for enhancers, the most useful features are CpG density and methylation autocorrelation (Figure 6B). Some features show strikingly different contribution in predicting promoters and enhances. For example, the feature of methylation variance is quite informative in promoter prediction, with information gain of 0.67 , while it has limited contribution when predicting enhancers, with information gain of 0.06 . This result suggests that a set of methylation-associated features are needed for predicting regulatory regions and these features have different predictive power for promoters and enhancers.

\section{Discussion}

Although low methylation has been found to be associated with regulatory regions $[16,21]$, we found that a low methylation level was not sufficient to predict regulatory regions, especially for enhancers. Additional features of $\mathrm{mCpG}$ sites were required to predict regulatory regions, and we were able to elucidate some of these features and successfully use them for prediction. For example, in regulatory regions, we found that a low methylation level extended across a range that was often longer than $1000 \mathrm{bp}$, and that the correlation of methylation levels between methylation sites was much stronger than outside of regulatory regions. Furthermore, we found a larger variation in methylation levels within regulatory regions compared to non-regulatory regions. Therefore, our work provides novel insights regarding the DNA methylation status in regulatory regions.

Since CpG islands are often lowly (or not at all) methylated and are considered to play an important role in gene regulation, the hypomethylated state is regarded to be important in positive gene regulation. In this study, we found that regulatory $\mathrm{mCpG}$ sites demonstrate distinct features beside hypomethylation. Specifically, we found that the methylation levels are highly correlated between neighboring $\mathrm{mCpG}$ sites in regulatory regions. Furthermore, when regulatory regions are inactive in other cell types, these methylation levels did not simply return to the background level but were instead hypermethylated, suggesting that in regulatory regions, a higher level of methylation is required to maintain an inactive state.
Correlation of the DNA methylation status of neighboring CpG sites $[25,26]$ has been previously observed; however, in these studies, the correlation was not analyzed in the context of regulatory regions. For example, Eckhardt et al. found an overall correlation between neighboring $\mathrm{CpG}$ sites in the human genome [25]. Our work revealed that the correlation primarily stems from the regulatory regions as the correlation in regulatory regions was much stronger than that in random genomic regions.

The overrepresented 8-mer motifs in regulatory regions are predicted as potential transcription factor binding sites. Our prediction suggested a distinct set of transcription factors might interact with these motifs in a methylation-dependent fashion since the overrepresented motifs had a higher GC content. While the chromatin structure was previously considered as one mediator of transcription factor-DNA interaction, our finding indicates that DNA methylation can also serve as a "switch" for protein-DNA interactions $[27,28]$. Indeed, the chromatin structure and DNA methylation can influence each other. For example, nucleosome occupancy can direct DNA methylation [29,30], and in some cases, DNA methylation can determine nucleosome occupancy [31].

In this study, we demonstrated that regulatory regions are predictable by their methylation patterns; however, our prediction did not perfectly separate regulatory and non-regulatory regions, especially at enhancers. One possible reason is that we did not have sufficient methylome datasets for the prediction model. Since these behaviors required methylation levels from multiple cell types, additional methylation data from a range of cell types should help to better describe these distinctive behaviors. We expect such datasets will become available in near future and will enable better prediction of the enhancers. Another possibility is that DNA methylation is also associated with other functional elements other than promoters and enhancers. For example, recent studies suggested that DNA methylation is also involved in alternative splicing regulation [32-34]. We need additional information to distinguish different types of functional elements to improve our prediction.

\section{Conclusion}

We proposed a set of novel methylation associated features that are informative to predict regulatory regions. These features greatly improve the prediction compared to the prediction solely based on methylation level. Our results suggest that the regulatory "grammar" is encoded in complex DNA methylation patterns and identification of these features will provide biological insight on the methylation-mediated gene regulation. 


\section{Methods \\ Data}

Human DNA methylation data as measured by bisulfite sequencing or Illumina 450k array available for 15 cell lines was obtained from SALK and ENCODE databases. The regulatory regions predicted from chromatin marks were downloaded from ENCODE database [35]. Gene expression data by RNA-Seq, available in 4 cell lines, was imported from SALK database [16]. RPKM (Reads per kilo base per million) values was used for transcripts' expression [36]. Transcription factor binding is based on ChiPseq or computational predictions [23,35]. Gene annotation from UCSC Genome database was used [37].

\section{CpG sites in the study}

The methylation level of a CpG site was measured as the ratio of the number of methylated cytosines to the total number of sequences on that position in the bisulfite sequencing data. To take into account only the sites with reliable measurement, we only consider the CpG sites covered with more than 4 sequences.

\section{Random region selection}

Only the mCpGs in the coding regions and the upstream regions of genes were considered, where the upstream region of a gene was defined as the $10 \mathrm{~kb}$ region upstream of the gene transcription start site (TSS). The immediate downstream gene was classified as the target gene for those identified mCpGs. To exclude the effect of differential methylation due to different genomic regions, we selected random regions with similar genomic positions as $\mathrm{mCpG}$ sites. For a regulatory region in the upstream region, we selected the corresponding random regions with the same relative distance to the TSS (Figure 7A).
Here the relative distance to the TSS was measured as the actual distance normalized by the total length of the coding region and the upstream region of the gene. For a regulatory region in an exon or an intron region, we selected the corresponding random regions with the same relative distance to the nearest boundary of the exon or intron of the target gene. Here the relative distance was normalized by the length of the exon or intron, depending on whether the region of interest is on exon or intron (Figure 7B). 1000 random region selections for each regulatory region were performed in this study.

\section{Autocorrelation of methylation profile}

Methylation level autocorrelation was computed as follows:

$$
r_{k}=\frac{\sum_{i=1}^{N-k}\left(x_{i}-\bar{x}\right)\left(x_{i+k}-\bar{x}\right)}{\sum_{i=1}^{N}\left(x_{i}-\bar{x}\right)^{2}}
$$

where $x_{i}$ is methylation level of a $\mathrm{mCpG}$ at position $i, x_{i+k}$ is methylation level of the mCpG distant $k$ nucleotides from position $i$, and $\bar{x}$ is mean methylation level of the $\mathrm{mCpGs}$ in all regions of interest. We considered the autocorrelation disappeared when the value reached 0.05 .

\section{CpG density and CG content}

CpG density was calculated as the number of CpGs in a region normalized by its length. CG content in a region was measured as the number of cytosines and guanines in the region normalized by its total length.

\section{Sequence motif discovery}

Only the 8-mer sequences with $\mathrm{CpG}$ in the center were considered. An 8-mer and its reverse complement were

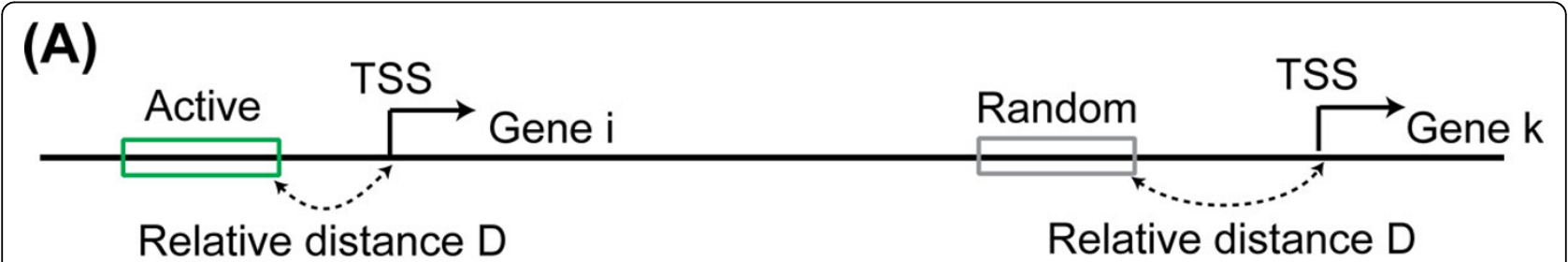

(B)

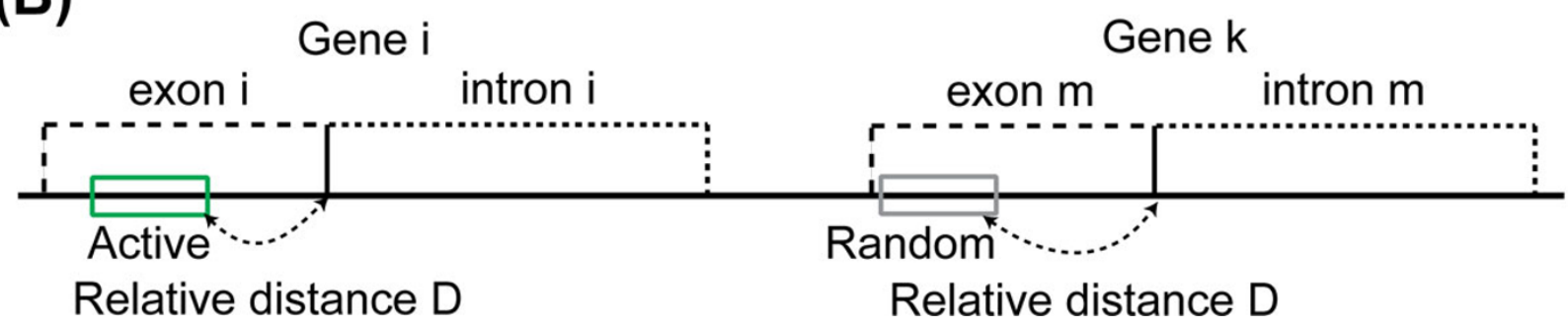

Figure 7 Schematic view of random region selection 
counted as the same motif. In theory, we have total 2080 possible 8 -mers with CpG in the center. For each motif, we calculated the occurrences of the motif in regulatory regions (either promoter or enhancer), and compared the occurrences of the same motifs in the random genomic regions. P-value for each 8-mers was calculated based on binomial distribution using the occurrence probability in the random regions as background probability.

$$
\text { pvalue }=1-\sum_{i=0}^{k}\left(\begin{array}{c}
n \\
i
\end{array}\right) p^{i}(1-p)^{n-i}
$$

where $p$ is probability that an 8 -mer is found in the random regions, and $k$ is the number of occurrences of the 8-mer of interest and $n$ is the number of all 8-mers in the regulatory regions. P-value was corrected for multiple testing using Bonferroni method.

\section{Regulatory region prediction}

Support Vector Machine (SVM) was used to predict regulatory regions based on the genomic features of the mCpGs in the regions. To apply SVM to our dataset, a number of features that represent the entities (regions) in the dataset should be identified and transformed into feature vectors, i.e. multi-dimensional vectors in which each element is a selected feature. SVM builds a set of hyperplanes that separate the entities into specified classes utilizing the provided feature vectors. In this research, the test data set for prediction includes the predicted regulatory regions and the same number of random regions generated as we described in the previous section. Five features were used to form the feature vector, including mean methylation level, mean methylation variance among 15 cell lines, mean methylation level autocorrelation between two mCpGs, CpG density, and 8-mer sequence motif $\mathrm{P}$-value around $\mathrm{mCpGs}$ in a genomic region. 10-fold cross validation was used to measure the prediction accuracy. In $\mathrm{k}$-fold cross validation, the dataset is randomly partitioned into $\mathrm{k}$ equal size of subsets. $\mathrm{k}-1$ subsets are used to train the prediction model and the remaining 1 subset is used to test the model. This cross validation process is repeated $\mathrm{k}$ times for each subset. For the SVM, polynomial kernel with the soft margin of 10 and the degree of 2 was used. The area under the ROC curve (AUC) was used to evaluate the prediction performance.

\section{Information gain}

Contribution of a feature $F$ in the classification for a sample set $S$ was calculated as the information gain of $S$ given the feature $F$, i.e., the difference between the entropy of $S$ without any feature knowledge and the entropy of $S$ given the feature $F$.

$$
I G(S, F)=H(S)-H(S \mid F)=H(S)-\sum_{v \in v a l u e s(F)} \frac{\left|S_{v}\right|}{|S|} H\left(S_{v}\right)
$$

$S$ is a set of samples, $F$ is a feature, $I G(S, F)$ is information gain for a set $S$ given a feature $F$, values $(F)$ is the set of all possible values of an attribute $F, S_{v}$ is the subset of $S$ that has value $v$ for an attribute $F$, and $H(S)$ is entropy of a set $S$ defined as follows.

$$
H(S)=-\sum_{i} p_{i} \log _{2}\left(p_{i}\right)
$$

where $p_{i}$ is the probability that a sample in $S$ is classified as class $i$. Discretization was adapted for continuous attributes.

\section{Additional material}

\section{Additional file 1: Figure S1. \\ Additional file 2: Figure S2. \\ Additional file 3: Figure S3. \\ Additional file 4: Figure S4. \\ Additional file 5: Figure S5.}

\section{Competing interests}

The authors declare that they have no competing interests.

\section{Authors' contributions}

WH performed bioinformatics analysis and drafted the manuscript. VO provided experimental data and drafted the manuscript. SM participated in its design, and drafted the manuscript. $\mathrm{HZ}$ participated in its design, and drafted the manuscript. JQ conceived the study, participated in its design, and drafted the manuscript. All authors read and approved the final manuscript.

\section{Acknowledgements}

This work was supported by funding from the National Institutes of Health [R01EY024580, R01GM111514 to J.Q., R21EY018703 to S.M, R01EY023188 to J. Q. and S.M.]; the Wilmer Core Grant [5P30EY001765 to J.Q.]; unrestricted funding from the Research to Prevent Blindness; the National Research Foundation of Korea grant [2011-0030810]; and the generosity of A. Nixon.

\section{Declarations}

The publication costs for this article were funded by the corresponding author.

This article has been published as part of BMC Genomics Volume 16 Supplement 7, 2015: Selected articles from The International Conference on Intelligent Biology and Medicine (ICIBM) 2014: Genomics. The full contents of the supplement are available online at http://www.biomedcentral.com/ bmcgenomics/supplements/16/S7.

\section{Authors' details \\ 'Department of Ophthalmology, Johns Hopkins School of Medicine, Baltimore, MD, 21205 USA. ${ }^{2}$ Department of Pharmacology and Molecular Sciences, Johns Hopkins School of Medicine, Baltimore, MD, 21205 USA. ${ }^{3}$ Center for High-Throughput Biology, Johns Hopkins School of Medicine, Baltimore, MD, 21205 USA. ${ }^{4}$ The Sidney Kimmel Comprehensive Cancer Center, Johns Hopkins School of Medicine, Baltimore, MD, 21025 USA. ${ }^{5}$ Data Science for Knowledge Creation Research Center, Seoul National University, Seoul, 151-742 Korea.}

Published: 11 June 2015 


\section{References}

1. Lee $D$, Karchin $R$, Beer M: Discriminative prediction of mammalian enhancers from DNA sequence. Genome Research 2011, 21:2167-2180.

2. Berman B, Pfeiffer B, Laverty T, Salzberg S, Rubin G, Eisen M, Celniker S: Computational identification of developmental enhancers: conservation and function of transcription factor binding-site clusters in Drosophila melanogaster and Drosophila pseudoobscura. Genome Biology 2004, 5:R61.

3. Pennacchio LA, Ahituv N, Moses AM, Prabhakar S, Nobrega MA, Shoukry M, Minovitsky S, Dubchak I, Holt A, Lewis KD, et al: In vivo enhancer analysis of human conserved non-coding sequences. Nature 2006, 444:499-502.

4. Berman BP, Nibu Y, Pfeiffer BD, Tomancak P, Celniker SE, Levine M, Rubin GM, Eisen MB: Exploiting transcription factor binding site clustering to identify cis-regulatory modules involved in pattern formation in the Drosophila genome. Proc Natil Acad Sci USA 2001, 99(2):757-762.

5. Johnson DS, Mortazavi A, Myers RM, Wold B: Genome-Wide Mapping of in Vivo Protein-DNA Interactions. Science 2007, 316(5830):1497-1502.

6. Park PJ: ChIP-seq: advantages and challenges of a maturing technology. Nature Reviews Genetics 2009, 10:669-680.

7. Landt SG, Marinov GK, Kundaje A, Kheradpour P, Pauli F, Batzoglou S, Bernstein BE, Bickel P, Brown JB, Cayting P, et al: ChIP-seq guidelines and practices of the ENCODE and modENCODE consortia. Genome Research 2012, 22:1813-1831.

8. Ernst J, Kheradpour P, Mikkelsen TS, Shoresh N, Ward LD, Epstein CB, Zhang X, Wang L, Issner R, Coyne M, et al: Mapping and analysis of chromatin state dynamics in nine cell types. Nature 2011, 473:43-51.

9. Thurman RE, Rynes E, Humbert R, Vierstra J, Maurano MT, Haugen E, Sheffield NC, Stergachis AB, Wang H, Vernot B, et al: The accessible chromatin landscape of the human genome. Nature 2012, 489(7414):75-82

10. Sheffield NC, Thurman RE, Song L, Safi A, Stamatoyannopoulos JA, Lenhard B, Crawford GE, Furey TS: Patterns of regulatory activity across diverse human cell-types predict tissue identity, transcription factor binding, and long-range interactions. Genome Research 2013, 23:777-788,

11. Ram O, Goren A, Amit I, Shoresh N, Yosef N, Ernst J, Kellis M, Gymrek M, Issner R, Coyne M, et al: Combinatorial Patterning of Chromatin Regulators Uncovered by Genome-wide Location Analysis in Human Cells. Cell 2011, 147(7):1628-1639.

12. Mikkelsen TS, Ku M, Jaffe DB, Issac B, Lieberman E, Giannoukos G, Alvarez P, Brockman W, Kim TK, Koche RP, et al: Genome-wide maps of chromatin state in pluripotent and lineage-committed cells. Nature 2007, 448:553-560

13. Pelizzola M, Ecker J: The DNA methylome. FEBS Letters 2011 585(13):1994-2000

14. Reik W, Dean W, Walter J: Epigenetic Repeogramming in Mammian Development. Science 2001, 293:1089-1093.

15. Baylin S: DNA methylation and gene silencing in cancer. Nature Clinical Practice Oncology 2005, 2:s4-s11.

16. Lister R, Pelizzola M, Dowen RH, Hawkins D, Hon G, Tonti-Filippini J, Nery JR, Lee L, Ye Z, Ngo QM, et al: Human DNA methylomes at base resolution show widespread epigenomic differences. Nature 2009, 462:315-322.

17. Meissner A, Gnirke A, Bell GW, Ramsahoye B, Lander ES, Jaenisch R: Reduced representation bisulfite sequencing for comparative highresolution DNA methylation analysis. Nucleic Acids Research 2005, 33:5868-5877.

18. Irizarry RA, Ladd-Acosta C, Wen B, Wu Z, Montano C, Onyango P, Cui H, Gabo K, Rongione M, Webster $M$, et al: The human colon cancer methylome shows similar hypo- and hypermethylation at conserved tissue-specific CpG island shores. Nature Genetics 2009, 41(2):178-186.

19. Touleimat N, Tost J: Complete pipeline for Infinium Human Methylation 450k BeadChip data processing using subset quantile normalization for accurate DNA methylation estimation. Future Medicine 2012, 4(3):325-341.

20. Burger L, Gaidatzis D, Schübeler D, Stadler MB: Identification of active regulatory regions from DNA methylation data. Nucleic Acids Research 2013, 41(16):e155

21. Stadler MB, Murr R, Burger L, Ivanek R, Lienert F, Schöler A, Nimwegen Ev, Wirbelauer C, Oakeley EJ, Gaidatzis D, et al: DNA-binding factors shape the mouse methylome at distal regulatory regions. Nature 2011, 480:490-495.

22. Lister R, Pelizzola M, Kida YS, Hawkins D, Nery JR, Hon G, AntosiewiczBourget J, O'Malley R, Castanon R, Klugman S, et al: Hotspots of aberrant epigenomic reprogramming in human induced pluripotent stem cells. Nature 2011, 471:68-75.

23. Pique-Regi R, Degner JF, Pai AA, Gaffney DJ, Gilad Y, Pritchard JK: Accurate inference of transcription factor binding from DNA sequence and chromatin accessibility data. Genome Research 2010, 21:447-455.

24. Choy MK, Movassagh M, Goh HG, Bennett MR, Down TA, Foo RS: Genomewide conserved consensus transcription factor binding motifs are hypermethylated. BMC Genomics 2010, doi: 10.1186/1471-2164-1111-1519.

25. Eckhardt F, Lewin J, Cortese R, Rakyan VK, Attwood J, Burger M, Burton J, Cox TV, Davies R, Down TA, et al: DNA methylation profiling of human chromosomes 6, 20 and 22. Nature Genetics 2006, 38:1378-1385.

26. Hodges E, Smith AD, Kendall J, Xuan Z, Ravi K, Rooks M, Zhang MQ, Ye K, Bhattacharjee $A$, Brizuela $L$, et al: High definition profiling of mammalian DNA methylation by array capture and single molecule bisulfite sequencing. Genome Research 2009, 19:1593-1605.

27. Spruijt CG, Gnerlich F, Smit AH, Pfaffeneder T, Jansen PWTC, Bauer C, Münzel M, Wagner M, Müller M, Khan F, et al: Dynamic Readers for 5-(Hydroxy) Methylcytosine and Its Oxidized Derivatives. Cell 2013, 152(5):1146-1159.

28. Hu S, Wan J, Su Y, Song Q, Zeng Y, Nguyen HN, Shin J, Cox E, Rho HS, Woodard C, et al: DNA methylation presents distinct binding sites for human transcription factors. elife 2013, 2:e00726.

29. Chodavarapu RK, Feng S, Bernatavichute YV, Chen PY, Stroud H, Yu Y, Hetzel JA, Kuo F, Kim J, Cokus SJ, et al: Relationship between nucleosome positioning and DNA methylation. Nature 2010, 466:388-392.

30. Felle $M$, Hoffmeister $H$, Rothammer J, Fuchs A, Exler JH, Längst G: Nucleosomes protect DNA from DNA methylation in vivo and in vitro. Nucleic Acids Res 2011, 39(16):6956-6696.

31. Portela A, Liz J, Nogales V, Setién F, Villanueva A, Esteller M: DNA

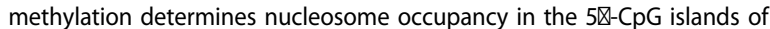
tumor suppressor genes. Oncogene 2013, doi:10.1038/onc.2013.1162.

32. Shukla S, Kavak E, Gregory M, Imashimizu M, Shutinoski B, Kashlev M, Oberdoerffer P, Sandberg R, Oberdoerffer S: CTCF-promoted RNA polymerase II pausing links DNA methylation to splicing. Nature 2011, 479:74-79.

33. Wan J, Oliver V, Zhu H, Zack D, Qian J, Merbs S: Integrative analysis of tissue-specific methylation and alternative splicing identifies conserved transcription factor binding motifs. Nucleic Acids Research 2013, 41(18):8503-8514.

34. Maunakea AK, Chepelev I, Cui K, Zhao K: Intragenic DNA methylation modulates alternative splicing by recruiting MeCP2 to promote exon recognition. Cell Research 2013, 23:1256-1269.

35. Raney BJ, Cline MS, Rosenbloom KR, Dreszer TR, Learned K, Barber GP, Meyer LR, Sloan CA, Malladi VS, Roskin KM, et al: ENCODE whole-genome data in the UCSC genome browser (2011 update). Nucleic Acids Research 2011, 39:D871-D875.

36. Mortazavi A, Williams BA, McCue K, Schaeffer L, Wold B: Mapping and quantifying mammalian transcriptomes by RNA-Seq. Nature Methods 2008, 5:621-628.

37. Meyer LR, Zweig AS, Hinrichs AS, Karolchik D, Kuhn RM, Wong M, Sloan CA, Rosenbloom KR, Roe G, Rhead B, et al: The UCSC Genome Browser database: extensions and updates 2013. Nuleic Acids Research 2012, 41(D1):D64-D69.

doi:10.1186/1471-2164-16-S7-S11

Cite this article as: Hwang et al:: Prediction of promoters and enhancers using multiple DNA methylation-associated features. BMC Genomics 2015 16(Suppl 7):S11. 